\title{
Ein Nachruf wird zum Krimi
}

\section{Eberhard Wolff}

Siehe zu diesem Thema auch das «Zu guter Letzt» auf S. 1406.
Im Juni starb 94-jährig der deutsche Ärztefunktionär Hans Joachim Sewering, Prof. Dr. med. Dr. h. c. Bei diesem Namen mögen dem einen oder anderen die Ohren klingeln. Neben unzähligen Vorstands- und Leitungsposten der deutschen bzw. bayerischen Ärzteschaft war Sewering seit 1973 für fünf Jahre Präsident der Bundesärztekammer. Er war Vorstand und Schatzmeister im Weltärztebund und vorgesehen, dessen Präsident, also der «höchste Arzt der Welt» zu werden. Internationale Proteste und eine Debatte um Sewerings Vorgeschichte machten dies zunichte, und so musste er 1993 seine Kandidatur zurückziehen. 1978 hatte ihn schon der deutsche Ärztetag zum Rücktritt gezwungen. In seinen bayerischen Ämtern konnte er sich noch bis 1992 halten.

Nicht nur, dass Sewerings Praxisführung Ungereimtheiten aufwies. Er war 1933 als 17-Jähriger der SS und im Folgejahr der NSDAP beigetreten. Zwischen 1943 und 1945 war er als Arzt des Tuberkulosekrankenhauses Schönbrunn bei Dachau verantwortlich für die Verlegung von Pfleglingen in die Heil- und Pflegeanstalt Egelfing-Haar. Mindestens neun Überweisungen tragen seine Unterschrift. Diese Einrichtung war damals als Zentrum der Krankentötungen in Oberbayern bekannt. Von diesen neun Patienten sind fünf in der Kinderfachabteilung oder den Hungerhäusern der «Euthanasie»-Anstalt ums Leben gekommen. Sewering bestritt zeitlebens, von den Vorgängen in Egelfing gewusst zu haben. Streng juristisch konnte es ihm auch nie endgültig nachgewiesen werden. Ein Ermittlungsverfahren gegen ihn wurde 1995 eingestellt.

Medizinhistoriker, die sich in den letzten Jahrzehnten intensiv mit dem Fall Sewering und seinem Umfeld befasst haben, gehen allerdings davon aus, dass es «kaum vorstellbar» sei, nichts davon gewusst zu haben. Dass eine solche Überweisung «einem Todesurteil nahe kam, war in Fachkreisen damals bekannt». Sewering habe die Menschen der NS-Euthanasie ausgeliefert.

All dies ist Geschichte, genauer gesagt: die Vorgeschichte des aktuellen Krimis post mortem. Und dieser beginnt mit der Nummer 28/29 des Deutschen Ärzteblatts (DÄB), der Standeszeitschrift der deutschen Ärzteschaft. Dort veröffentlichten Sewerings Nachfolger, der deutsche Ärztekammerpräsident Prof. JörgDietrich Hoppe und sein Vorgänger, Prof. Karsten Vilmar, einen Nachruf auf Sewering unter dem Titel «Gestalter im Dienst der Ärzteschaft». Darin wird gewürdigt, wie sich Sewering «für die Belange der Ärzte- schaft und für eine gute Versorgung der Patienten eingesetzt» habe. Die Druckseite kulminiert in der Feststellung, Sewering habe sich «um die Wahrung ethischer Normen ärztlichen Handelns verdient gemacht». Die Autoren gehen in ihrem Nachruf mit keinem Wort auf die diskutierten Verstrickungen des Verstorbenen in die NS-Euthanasie ein. Und dies, obwohl der Umgang der organisierten deutschen Ärzteschaft mit der überaus problematischen Rolle der Ärzte im Nationalsozialismus eine eigene Geschichte hat, die von Verdrängung, Abwehr, insbesondere in den letzten Jahren aber auch von aktiver Aufarbeitung, Offenheit und Engagement gezeichnet ist. Ein heikles Politikfeld, bis heute.

Widerspruch liess allerdings nicht lange auf sich warten. 80 Medizinhistoriker, organisiert vom Münchner Medizinhistoriker und Sewering-Fall-Forscher Dr. Gerrit Hohendorf, schickten Ende Juli einen Brief an die Verfasser und als Leserbrief an das DÄB, in dem sie in aller Form kritisierten, dass der Nachruf die NSVergangenheit Sewerings verschweige. Dies sei für sie «schwer nachvollziehbar». Sie wollten nicht «persönlich anklagen oder verurteilen», doch sei ihnen die «Ergänzung historischer Fakten wichtig». Während der öffentlichen Debatte um Sewerings Rolle im Nationalsozialismus wäre «ein Wort des Bedauerns» von seiner Seite «nicht zuviel gewesen». Zur «Wahrung ethischer Normen ärztlichen Handelns», um die sich Sewering den Autoren des Nachrufs zufolge verdient gemacht habe, gehöre es auch, historische Verantwortung zu übernehmen.

\section{«Widerspruch liess allerdings nicht} lange auf sich warten»

$\mathrm{Zu}$ diesem Zeitpunkt waren die Medien aber bereits auf die Geschichte aufgesprungen. Gegenüber dem Westdeutschen Rundfunk verteidigte sich der Ärztekammer-Sprecher, dass nur die standespolitische Tätigkeit gewürdigt werden sollte, dass die Vorwürfe gegen Sewering nicht letztlich geklärt seien und dass ein Nachruf nicht der Ort sei, dies zu diskutieren. Hoppe selbst meinte gegenüber der «Frankfurter Rundschau», er hätte den Text gemäss dem Motto «De mortuis nil nisi bene» verfasst, nach dem man über die 
Toten nichts als Gutes sagen solle. Die Ärztekammer und auch er seien seit Jahren bemüht, «die Rolle und Verantwortung der Ärzteschaft im Nationalsozialismus aufzuarbeiten».

Auch der Chefredakteur des DÄB Heinz Stüwe erklärte sich in den Medien. Er wolle den Leserbrief nicht abdrucken, weil man zu Nachrufen keine Leserbriefe veröffentliche. Auch sei er verärgert über die selektive Wahrnehmung der Briefschreiber. Sewerings Vorgeschichte sei an anderer Stelle im DÄB bereits angesprochen worden. In der Tat: Stüwe selbst hatte die Hälfte einer ersten 30-Zeilen-Meldung über Sewerings Ableben in der Nr. 26 des DÄB der problematischen Seite des Verstorbenen gewidmet und skizziert, warum Sewering «eine der umstrittensten Personen der deutschen Ärzteschaft» (Süddeutsche Zeitung) gewesen sei. Ausserdem habe das DÄB in anderen Artikeln über Sewerings problematische Vergangenheit geschrieben. Zwei der vier Artikel stammen von Stüwes Chefredakteur-Vorgänger Norbert Jachertz.

\section{«Wir werden den Verstorbenen stets in Erinnerung behalten»}

Noch vor Redaktionsschluss des Artikels in der Frankfurter Rundschau änderte Stüwe seine Meinung: Der Leserbrief solle jetzt doch abgedruckt werden (erschienen in Nr. 31/32). Auch der Vorsitzende des Fachverbands Medizingeschichte, Prof. Heiner Fangerau, hatte sich für den Abdruck eingesetzt.

Dass es auch anders ging, zeigten die Kollegen von der Bayerischen Landesärztekammer. Deren Präsident Prof. Hans Hellmut Koch verfasste nämlich in der Landes-Standeszeitschrift «Bayerisches Ärzteblatt» ebenfalls einen Nachruf auf Sewering. Der beginnt mit einer Auflistung aller standespolitischen Ämter Sewerings. Das letzte Drittel der knappen Druckseite spricht die Kritik und Vorwürfe gegen Sewering vorsichtig, aber offen an. Und wiederum ist der Schlusssatz bezeichnend: «Wir werden den Verstorbenen stets in Erinnerung behalten.» Ist da bewusst ein Adjektiv weggelassen worden?

Man muss auf der Suche nach anderen Wegen allerdings gar nicht so weit in die Ferne schweifen. Das DÄB selber betreibt nämlich neben seinem «Flaggschiff» unter anderem auch fünf Blogs. Einer davon heisst «Lesefrüchtchen» und wird vom früheren DÄBChefredakteur Norbert Jachertz geschrieben. Und die- ser bloggte unter dem Titel «Hoch gestiegen - tief gefallen» nicht unkritisch über den früher sogenannten «König von Bayern» und spürte sogleich das fehlende Adjektiv «dankbar» im bayerischen Nachruf auf.

\section{Postskriptum}

Der Autor hat in den 1990er Jahren in einer Forschergruppe im Auftrag der Bundesärztekammer die Geschichte der Ärztlichen Standesvertretung in Deutschland mit aufgearbeitet und stellt fest, dass die damalige Arbeit völlig unbeeinflusst geschehen konnte. Im Gegenteil wurde eine «kritische» Aufarbeitung gewünscht.

\section{Literatur}

- Nachruf im DÄB (Nr. 28-29/2010) auf Sewering: www.aerzteblatt.de/v4/archiv/artikel. asp? src=heft\&id=77604

- Meldung des Chefredakteurs im DÄB (Nr. 26/2010) über den Tod Sewerings: www.aerzteblatt.de/v4/ archiv/artikel.asp?src=suche $\& \mathrm{p}=$ sewering $\& \mathrm{id}=77311$

- Nachruf im Bayerischen Ärzteblatt (Ausgabe Juli-August 2010) auf Sewering: www.blaek.de/ presse/aerzteblatt/2010/BAB_0710_392_393.pdf

- Erste Meldung im DÄB-Blog «Lesefrüchtchen» (25.6.2010): www.aerzteblatt.de/v4/blogs/beitrag. asp? $\mathrm{id}=41737 \& \mathrm{p}=$ gefallen + jachertz

- Zweite Meldung im DÄB-Blog «Lesefrüchtchen» (15.7.2010): www.aerzteblatt.de/blogs/42000/ Nochmals_zu_Sewering_Ein_Woertchen_fehlt.htm

- Bericht im Westdeutschen Rundfunk (WDR 5, 26.7.2010): www.wdr.de/themen/kultur/medien/ aerzteblatt/index.jhtml?rubrikenstyle=kultur

- Bericht in der Frankfurter Rundschau (29.7. 2010): www.fr-online.de/politik/blinder-fleck-im-leben-desaerztefunktionaers/-/1472596/4517028/-/index.html

- Bericht der Frankfurter Allgemeinen Zeitung vom 28.7.2010 (nicht im open access der Zeitung): http://germanmediawatch.posterous.com/

- Leserbrief der 80 Medizinhistoriker: www.aerzteblatt.de/v4/archiv/artikel.asp?src= suche\&p=hohendorf\&id=77851

- Im Online-Archiv des DÄB weitere Artikel zum Thema unter Stichwort «sewering». Literaturhinweise zu medizinhistorischen Arbeiten über den Fall Sewering am Ende des o.g. Leserbriefs.

- Artikel im Magazin «Focus» über Sewering und die Euthanasie-Fälle (1995): www.focus.de/politik/ deutschland/euthanasie-die-mordbilanz-von-1be aid_153037.html

- Buch über die Geschichte der Ärztlichen Standesvertretung in Deutschland im Auftrag der Bundesärztekammer: Jütte R (Hrsg.). Geschichte der deutschen Ärzteschaft. Organisierte Berufs- und Gesundheitspolitik im 19. und 20. Jahrhundert. Köln: Deutscher Ärzte-Verlag; 1997. 